\title{
Über die „keimungshemmenden“ Stoffe aus Früchten
}

\author{
Von Karl PaEch \\ Aus dem Botanischen Institut der Universität Tübingen \\ (Z. Naturforschg. 4b, 46-50 [1949]; eingegangen am 14. Januar 1949)
}

\begin{abstract}
Die Vorstellung von der natïrlichen Bedeutung keimungshemmender Stoffe in saftigen Früchten und Samenhüllen ist bis jetzt noch nicht widerspruchsfrei ausgebaut. In der Schleimhülle von Kakao-Samen lassen sich Substanzen nachweisen, die anfangs die Keimzahl bei Lepidium-Samen, später, aus vollreifen Kakao-Früchten, nur noch die Länge der Primärwurzeln von Lepidium-Keimlingen herabsetzen. Trotz der Anwesenheit solcher „Blastokoline“ keimen die Kakao-Samen in der Frucht und im Keimbett aus. Die Art und Menge solcher Stoffe, einerseits in keimenden und andererseits in nichtkeimenden KakaoSamen, läßt sich mit dem Lepidium-Test nicht als verschieden erweisen. Sie können deshalb nicht für das normale Unterbleiben der Samenkeimung in den Kakao-Früchten verantwortlich sein.
\end{abstract}

G enuine keimungshemmende Substanzen, die dafür verantwortlich sein sollen, daß Samen in saftreichen Früchten nicht auskeimen, sind schon vor langer Zeit entdeckt ${ }^{1}$, extensiv studiert (Übersicht bei $\mathrm{Molisch}{ }^{2}$ und bei $\mathrm{Ruge}^{3}$ ), mit einem besonderen Namen belegt ${ }^{4}$ und neuerdings auch chemisch charakterisiert worden ${ }^{5}$, so daß man geneigt sein könnte, sie zum gesicherten Bestand an pflanzlichen Hormonen zu rechnen. Um so überraschter ist man, bei genauerer Überlegung einige recht offensichtliche Mängel und Unzulänglichkeiten in der Vorstellung einer Keimungshemmung durch Inhaltsstoffe der Fruchthülle zu finden.

1. Solche Hemmstoffe sind u. a. auch im Fleisch von Äpfeln, Birnen. Paprikafrüchten usw. nachgewiesen worden. Der Bau dieser Früchte macht jedoch eine Einwirkung nichtflüchtiger Stoffe aus dem Fruchtfleisch auf die Samen sehr unwahrscheinlich. Flüchtige keimungshemmende Substanzen sind bisher ausgerechnet im Pericarp von Gurken gefunden worden ${ }^{6}$.

2. Die Stärke solcher Hemmstoffe wird seit W i es n e r vornehmlich mit Samen von Lepidium sativum ausgetestet und nur selten an den Samen derjenigen Früchte erprobt, die die „Blastokoline“ liefern. Die eigenen Samen der Frucht keimen denn auch mehr oder weniger regelmäßig aus,

1 J. W i e s n e r, S.-B. Akad. Wis: Wien, Mathem.naturwiss. Kl. Abt. I, 103, 401 [1894].

${ }^{2}$ H. II o l i s c h, Der Einfluf einer Pflanze auf die andere. Jena 1937.

${ }^{3}$ L. R u g e, Z. Bot. 33, 529 [1939]. wenn Lepidium-Samen stark oder völlig gehemmt sind (s. Funke ${ }^{7}$ und u.).

3. Die Natur der Hemmstoffe wird meist als nicht artspezifisch beschrieben, und doch können häufig aus lebhaft keimenden Samen oder Samenteilen Stoffe extrahiert werden, die nur auf die Samen anderer Arten hemmend wirken. Besonders ausgeprägt ist diese ,Fremdwirkung“ auch bei Samen bzw. Früchten, die ätherische Öle enthalten und keimen, ohne diese Substanzen abzubauen oder zu verändern, während bei Wicke, Weizen, Raps u. a. gerade ätherische Öle die Keimzahl herabsetzen und das Wurzelwachstum stark verzögern ${ }^{8}$. Eine biologische Bedeutung solcher Hemmstoffe für die eigenen Samen fehlt hier also ganz offensichtlich.

4. Obwohl die natürliche Funktion der fraglichen Stoffe in einer Verhinderung des Auskeimens der Samen in den Früchten bestehen soll, wird ,die Blastokolinwirkung durch die prozentuale Hemmung des Längenwachstums der Radicula gegenüber der Kontrolle ausgedrückt" ". Solche Hemmstoffe könnten also bestenfalls erklären, daß gekeimte Samen in den Früchten nur gehemmt weiterwachsen. Das Durchstoßen der Testa und damit die allein entscheidende Zahl der ..Keimprozente" wird häufig gar nicht registriert,

${ }_{4}^{4} \mathrm{~A}$. K ̈̈ c k e m a $\mathrm{n} n$, Ber. dtsch. bot. Ges. 52, 523 [1934].

5 R. K u h n u. Mitarbb., Naturwiss. 31, 468 [1943].

6 E. K a u f m a n n, Planta 33, 516 [1943].

7 H. F u n k e, Beih. Bot. Zbl. 59 A, 235 [1939].

8 W. Si g m und, Biochem. Z. 146, 389 [1924].

${ }^{9}$ F. II o e w us, Natur und Volk 77, 98 [1947]. 
jedenfalls nicht der Auswertung zugrunde gelegt. Das Wurzelwachstum wird jedoch auch von Wuchsstoffen (z. B. 3-Indolylessigsäure) gehemmt, und es erscheint deshalb ungereimt, die durch Hemmung des Wurzelwachstums bestimmten ..Blastokoline“"als „Antagonisten der Wuchsstoffe“ hinzustellen.

5. Solche keimungshemmende Substanzen werden nicht nur in Früchten, sondern praktisch in beliebigen anderen pflanzlichen Organen gefunden. Es erscheint willkürlich, ihnen gerade in Früchten eine bestimmte biologische Bedeutung zuzusprechen. Auf der anderen Seite handelt es sich nicht um spezifische Stoffe, denn organische Säuren ${ }^{10}$, Rohrzucker ${ }^{11}$ und andere allgemeine Inhaltsstoffe pflanzlicher Zellen hemmen in ähnlicher Weise wie die zunächst chemisch nicht identifizierten ,.Blastokoline“. Cumarin und andere ungesättigte Laktone, denen man eine spezifische Bedeutung in diesem Zusammenhang zugesprochen hat, zeichnen sich mit ihrer Wirkung auf manche Objekte vor anderen allgemein verbreiteten Verbindungen nicht aus ${ }^{12}$. Andererseits ist Ascorbinsäure, eine $\gamma$-Laktonverbindung, noch nie als keimungshemmend bezeichnet worden.

6. Die Wirkung ,,der" keimungshemmenden Stoffe aus Fruchtfleisch soll einerseits durch Kochen vernichtet ${ }^{11}$, andererseits durch Kochen verstärkt ${ }^{7}$ werden. Die betreffenden Substanzen sollen das eine Mal alkohollöslich ${ }^{6}$, das andere Mal alkoholfällbar ${ }^{11}$ sein. Jeweils sind die dazu passenden, einander also widersprechenden Schlüsse gezogen worden.

Diese und weitere Unstimmigkeiten lassen die Funktion chemisch bestimmter Blastokoline, die als Antagonisten der Wuchsstoffe und damit als „Wachstumsregulatoren" in das normale Geschehen eingreifen sollen, noch recht fragwürdig erscheinen. $\mathrm{Ob}$ und in welcher Weise keimungshemmende Substanzen eine Rolle im natürlichen Entwicklungsablauf der Samenpflanzen spielen, muß also erst durch Analyse weiterer Einzelfälle klargestellt werden.

\section{Eigene Versuche}

Von Kakao-Samen (Theobroma cacao) ist bekannt, daß sie oft in der Frucht auskeimen, ohne

10 W. A. Tet j u r w, Planta 32, 211 [1941].

11 H. Op p enh e ime r, S.-B. Akad. Wiss. Wien, Mathem.-naturwiss. Kl. Abt. I, 131, 279 [1922].

12 R. L. We in t r a u b, Smithsonian Miscell. Coll. 107, Nr. 20 [1948]. daß die Bedingungen, unter denen dies geschieht, genauer beschrieben sind. Da die Samen zudem von einer dicken Schleimschicht eingehüllt sind, erschien es lohnend, an diesem Objekt einmal nach Hemmstoffen und deren Verhalten bei der Keimung zu fahnden.

Ein stattlicher Kakaobaum in den Tübinger Gewächshäusern trägt regelmäßig einen reichlichen Fruchtbehang mit voll keimfähigen Samen. Im Sommer 1948 reiften an diesem Baum 25 ausgewachsene Früchte. Aus einem Teil der Samen sind inzwischen kräftige Jungpflanzen gezogen worden. Ein anderer Teil der Früchte stand zu diesen Versuchen zur Verfügung. Die Keimfähigkeit der Kakao-Samen erhält sich nur wenige Wochen nach der Ernte der Früchte.

In Petrischalen von 9,5 cm innerem Durchmesser, in denen sich eine dreifache Lage von Filtrierpapier befand, wurden $7 \mathrm{~cm}^{3}$ Extrakt bzw. dest. Wasser bei den Kontrollen gegeben. Auf das Fließpapier kamen, möglichst gleichmäßig verteilt, 100 ausgelesene Samen einer Handelssorte von Lepidium sativum. Die ersten Serien mußten, da ein Thermostat nicht zur Verfügung stand, bei Zimmertemperatur, die im Mai und Juni zwischen Tag und Nacht in recht weiten Grenzen schwankte, gehalten werden. Die späteren Reihen keimten im Thermostaten bei $24^{\circ} \mathrm{C}$. Nach $40 \mathrm{Stdn}$. wurde die Anzahl der Samen festgestellt, bei denen die Radicula makroskopisch sichtbar die Testa durchbrochen hatte (= Keimprozent), und die Länge der Keimwurzel gemessen. Die Zahl der in den Kontrollschalen gekeimten Samen wurde als $100 \%$ gesetzt; von den 100 ausgelegten Samen waren dies zwischen 85 und 90 Stück.

Am 12. Mai wurden die schleimigen Samenhüllen von 20 Kakao-Samen einer noch nicht ausgereiften Frucht nach dem Abschaben mit $50 \mathrm{~cm}^{3}$ dest. Wasser extrahiert. Der Extrakt hatte nach dem Abpressen durch ein Filtertuch ein $p_{\mathrm{H}} \mathrm{zwi}$ schen 2 und 3 (Merckscher Universalindikator). Er wurde mit Natronlauge neutralisiert $\left(p_{\mathrm{H}}=7\right)$. Die Keimversuche mit Lepidium-Samen sind in Tab. 1 zusammengestellt.

\begin{tabular}{|c|c|c|c|c|c|}
\hline $\begin{array}{c}\text { Ver- } \\
\text { dünnung } \\
\text { des } \\
\text { Extraktes }\end{array}$ & $\begin{array}{c}\text { Keim- } \\
\text { prozent } \\
\text { Kontrolle } \\
=100)\end{array}$ & $\begin{array}{c}\text { im Durchschnitt } \\
\text { im }\end{array}$ & $\begin{array}{c}0_{0} \text { der } \\
\text { Kontrolle }\end{array}$ & $\begin{array}{c}\text { Minimum } \\
\text { mm }\end{array}$ & $\begin{array}{c}\text { Maximum } \\
\text { mm }\end{array}$ \\
\hline unverdünnt & 0 & - & - & - & - \\
$1: 10$ & 86 & 3,3 & 36,6 & 0,5 & 8 \\
$1: 100$ & 100 & 6,3 & 70,0 & 0,5 & 15 \\
Kontrolle & 100 & 9,0 & 100 & 1,0 & 16 \\
\hline
\end{tabular}

Tab. 1. Extrakt aus den Samenschleimen einer un. reifen Kakao-Frucht, geprüft mit Lepidium-Samen 
Die im unverdünnten Extrakt nicht gekeimten Samen wurden 2 Stdn. mit dest. Wasser gewässert und keimten dann zum gleichen Prozentsatz wie die Kontrollen aus; eine Schädigung hinterblieb nicht. Keimversuche mit Kakao-Samen dieses Reifegrades wurden nicht angestellt, weil sie nach den Erfahrungen der Gärtner noch nicht keimfähig sind. In einer nächsten Saison müßte allerdings noch geklärt werden, ob nach Entfernen der Schleimschicht und nach dem Auswässern die unreifen Samen nicht doch zum Keimen zu bringen sind. Die Wahrscheinlichkeit dafür ist allerdings sehr gering. Die mangelnde Keimfähigkeit dürfte in diesem Zustand durch innere Ursachen (Unreife des Embryo o. ä.) bedingt sein.

In den weiteren Versuchen wurden jeweils zehn Kakao-Samen mit $30 \mathrm{~cm}^{3}$ dest. Wasser extrahiert. Die Schleimhülle der Samen wurde dabei so vollständig wie möglich mit einem Hornspatel abgeschabt. Die Ergebnisse sind in Tab. 2 zusammengefaßt. Die Extrakte wurden auch hierbei vor den Versuchen stets auf $p_{\mathrm{H}} 7$ gebracht.

\begin{tabular}{|c|c|c|c|c|c|c|c|c|}
\hline \multirow[b]{2}{*}{$\mathrm{Nr}$. } & \multirow[b]{2}{*}{ Datum } & \multirow{2}{*}{$\begin{array}{c}\text { Ur- } \\
\text { sprüng } \\
\text { liches } \\
p_{\mathrm{H}} \\
\text { etwa }\end{array}$} & \multicolumn{2}{|c|}{$\begin{array}{c}\text { Unverdünnter } \\
\text { Extrakt }\end{array}$} & \multicolumn{2}{|c|}{$\begin{array}{c}\text { Verdünnung } \\
1: 1\end{array}$} & \multicolumn{2}{|c|}{$\begin{array}{c}\text { Verdünnung } \\
1: 10\end{array}$} \\
\hline & & & 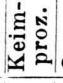 & $\begin{array}{l}\text { Wurzel- } \\
\text { länge } \% \\
\text { der Kontr. }\end{array}$ & 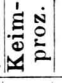 & $\begin{array}{c}\text { Wurzel- } \\
\text { länge } \\
\% / 0\end{array}$ & 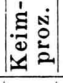 & $\begin{array}{c}\text { Wurzel- } \\
\text { länge } \\
0 / \%\end{array}$ \\
\hline 1. & 8. Juni & 4 & 100 & 10 & - & - & 100 & 70 \\
\hline 2. & 6. Aug & $\left.5^{*}\right)$ & 100 & 1 & 100 & 25 & 100 & 67 \\
\hline 3. & & $\left.5^{*}\right)$ & 100 & 12 & 100 & 24 & 100 & 65 \\
\hline
\end{tabular}

* Die Schleimschicht der Samen vor der Extraktion ergab, mit dem Indikatorpapier geprüft, ein $p_{\mathrm{H}}$ von etwa 3.

Tab. 2. Extrakte aus den Samenschleimen von KakaoFrüchten verschiedener Erntedaten, geprüft mit Lepidium-Samen.

Die Kakao-Früchte waren Anfang Juni soweit gereift, daß die Samen, in die Erde gebracht, ohne weiteres auskeimten (s. u.). Im August und September handelte es sich um ausgesprochen überreife Früchte. Die Frucht, die am 6. August geerntet wurde, war an einer Stelle von Schimmelpilzen befallen. Von den 18 Samen hatten 10 in der Frucht 5-10 $\mathrm{mm}$ lange Keimwurzeln getrieben. Die mit dem Lepidium-Test bestimmbaren ,.Blastokoline“ hatten sich jedoch gegenüber den 2 Monate vorher an ungekeimten Samen ermittelten nicht vermindert. Das Auskeimen in der Frucht kann also kaum durch das Verschwinden der Hemmstoffe bedingt sein. Am 27. September wurden 2 Früchte geöffnet. Das Fruchtfleisch war mürber als in den früheren Reifestadien. Eine der beiden Früchte war wieder von Schimmelpilzen befallen. Von 19 Samen war einer ausgekeimt. Die Hemmstoffe zeigten, mit Lepidium geprüft. keine Veränderung. Ein Vergleich der Schleimextrakte reifer Samen mit dem Extrakt aus unreifen ergibt, daß hier bei einer Hemmung des Wurzelwachstums auf $36 \%$ der Kontrolle schon eine merkliche Herabsetzung der Keimprozente erfolgt (Tab.1, Verdünnung 1:10), während später, trotz einer Wurzellänge von durchschnittlich nur $15 \%$, die Keimung selbst nicht mehr eingeschränkt ist (Tab. 2, unverdünnter Extrakt), wobei die nicht ganz gleichen Ausgangskonzentrationen eine noch weitere Kluft verbergen. Im übrigen erscheint es so, als würde mit der Reifung die Konzentration der fraglichen Stoffe vermindert, was aber nichts Spezifisches bedeutet, sondern für die verschiedensten Inhaltsstoffe reifender Früchte zutrifft, z. B. für organische Säuren.

Die Keimversuche mit Kakao-Samen ergaben folgendes: Anfang Juni wurden neben frisch der Frucht entnommenen Samen solche ausgelegt, die, den Gepflogenheiten der Gärtner entsprechend, 3 Tage bei Zimmertemperatur zum Austrocknen der Schleimschicht gelegen hatten. Irgendein Unterschied im Keimverhalten gegenüber den frisch entnommenen Samen zeigte sich nach dieser Vorbehandlung nicht. Alle 15 Samen (5 getrocknete und 10 frische) hatten nach 14 Tagen in großen Schalen (mit feuchtem Fließpapier ausgelegt), die im Warmhaus bei etwa 18 bis $20^{\circ}$ standen, Keimwurzeln von durchschnittlich $2 \mathrm{~cm}$ Länge getrieben, die zumeist positiv geotrop gekrümmt waren. Soweit die Wurzelspitzen das feuchte Fließpapier berührten, in das aus der Schleimhülle der Samen Stoffe diffundiert sein mußten, wurden sie schwarz; sie erschienen geschädigt. Obwohl die Schleimschicht, mit Lepidium geprüft, ,.Blastokoline“ enthielt, keimten die Kakao-Samen innerhalb dieser Hülle vollzählig aus. Dabei umgaben die fraglichen Stoffe die Kakao-Samen in wesentlich höherer Konzentration als im Extrakt, der den Lepidium-Samen gèboten wurde. Auch bei Misteln hemmt der Schleimextrakt aus Beeren, deren Samen innerhalb des Schleimes ausgekeimt sind, noch die Keimung von Lepidium-Samen ${ }^{\top}$.

Ende September wurden 10 Kakao-Samen, deren Schleimhüllen abgeschabt worden waren, neben 


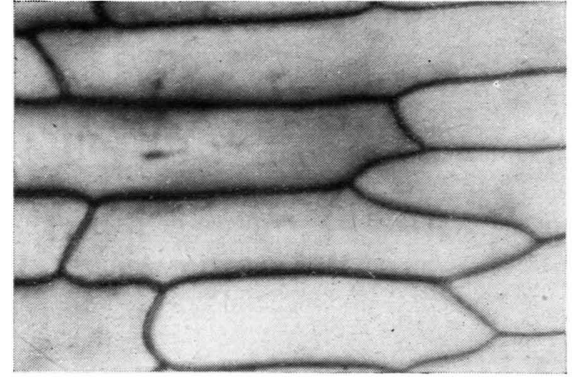

Abb. 1. Oberepidermiszellen der Schuppenblätter von Allium Cepa nach 12-stdg. Aufenthalt in einer Mischung von $5 \mathrm{~cm}^{3}$ 0,01-proz. Methylenblau $+1 \mathrm{~cm}^{3} 0,1-m . \mathrm{KNO}_{3}$ : reine Membranfärbung.

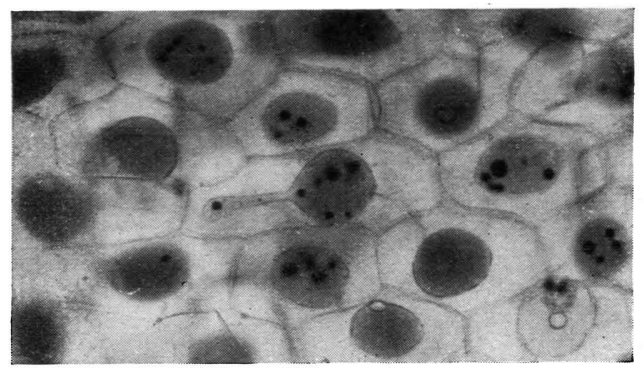

Abb. 2. Unterepidermiszellen der Schuppenblätter von Allium Cepa nach 12-stdg. Aufenthalt in einer Mischung von $5 \mathrm{~cm}^{3} 0,01$-proz. Methylenblau $+1 \mathrm{~cm}^{3} 0,1-\mathrm{m}$. $\mathrm{Ca}\left(\mathrm{NO}_{3}\right)_{2}$ : reine Vakuolenfärbung. Zellen vor der Aufnahme mit $1-m$. $\mathrm{Ca}\left(\mathrm{NO}_{3}\right)_{2}$ plasmolysiert. Die Plasmolyse bedingte in einigen Zellen die Bildung von stärker gefärbten Entmischungstropfen.

H.Schanderl, G.La uff $\dagger$ und H.Becker, Studien über die Mycetom- und Darmsymbionten der Aphiden (S.50)
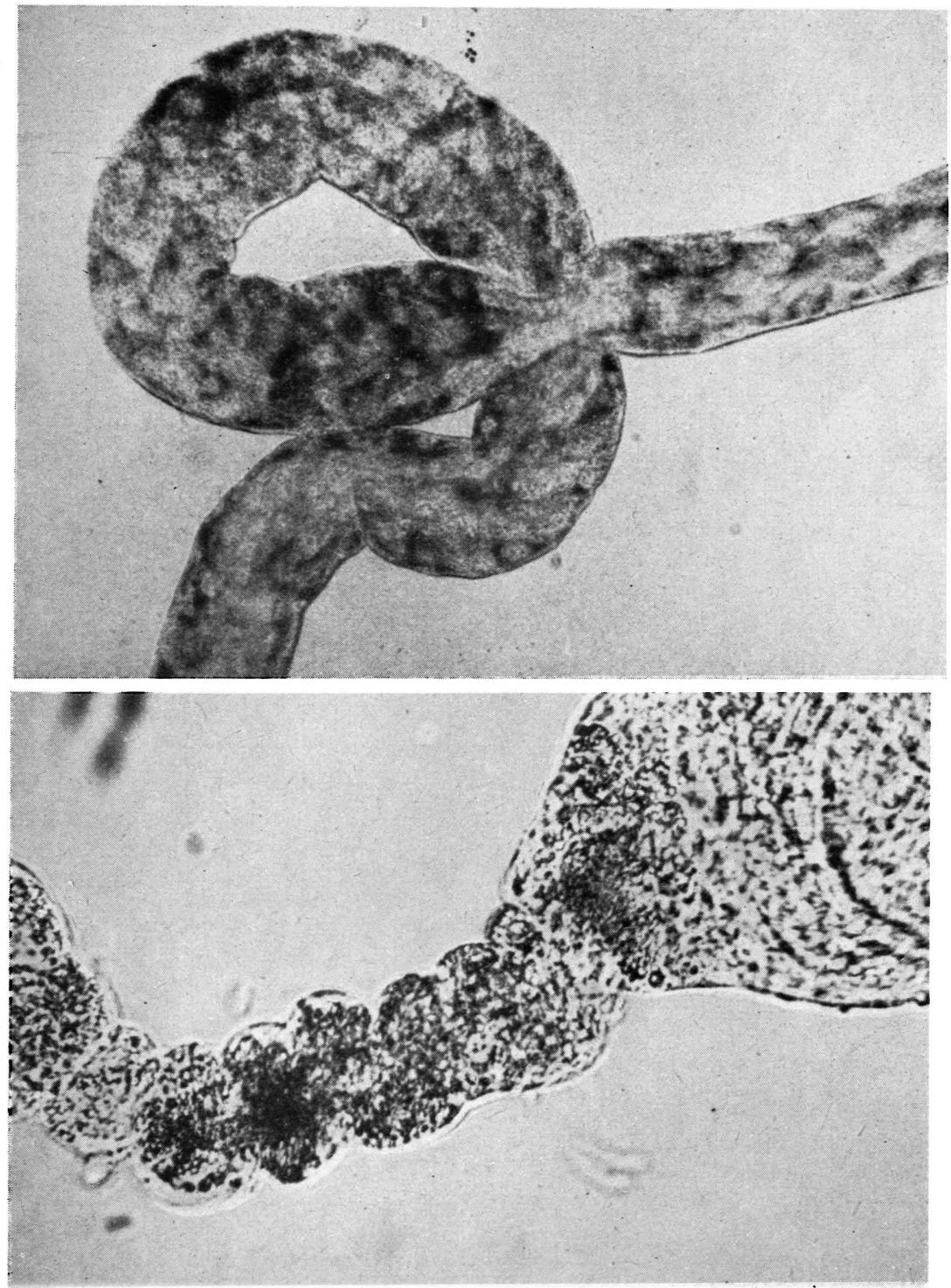

Abb. 1. Mitteldarm von Myzodes persicae Sulzer. Man erkennt noch gut die Abgrenzungen der Darmepithelzellen mit ihrem körnigen Inhalt. Vergr. etwa 500/1.
Abb. 2. Magen und Darm von Phylloxera vastatrix. Auch hier sind die Darmepithelzellen mit Granulationen gefüllt, und zwar bis zum Magen. Dieser selbst ist frei davon. Vergr. etwa 500/1. 

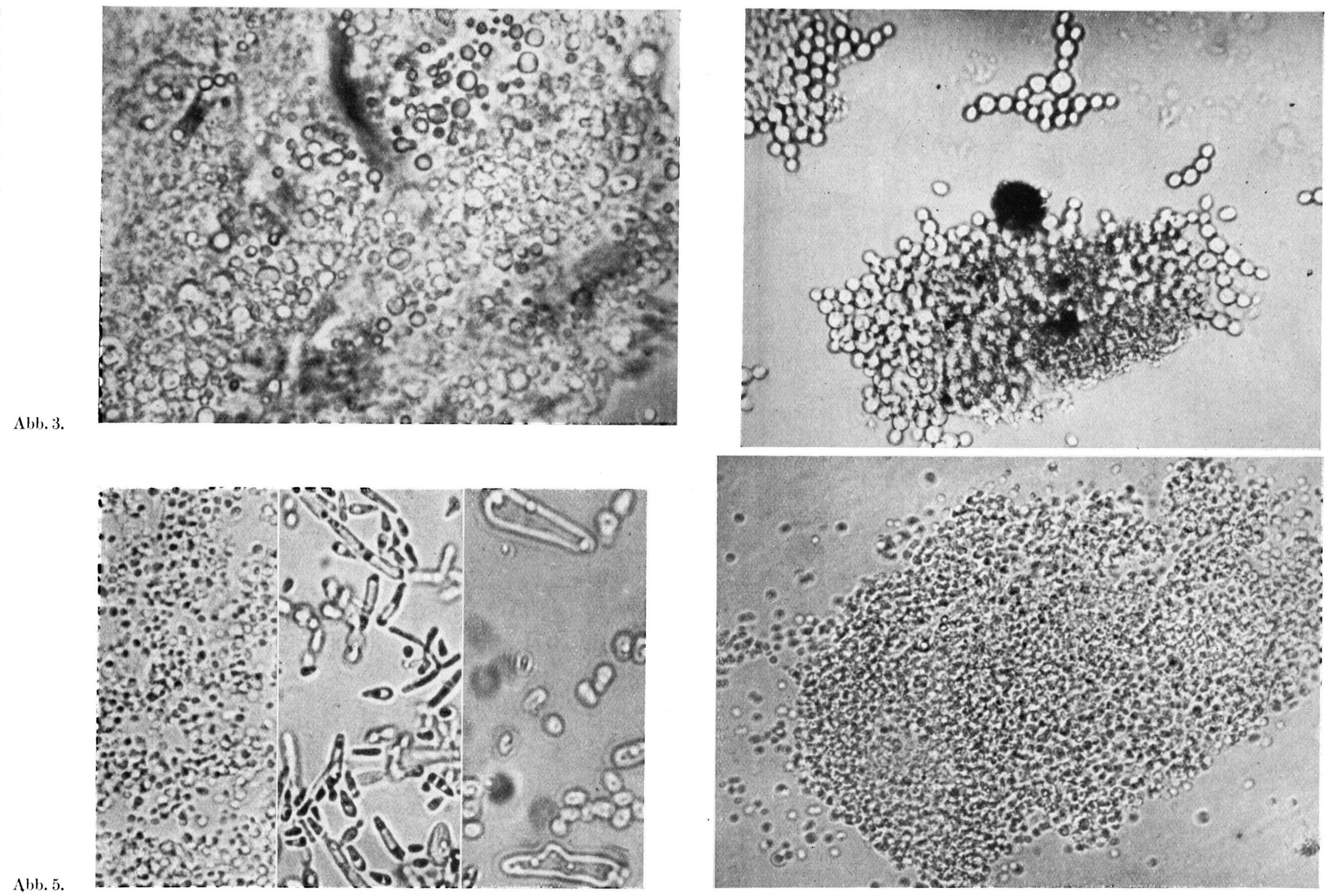

Abb. 4 .

Abb. 5. a

b c

Abb. 3. Drei Darmepithelzellen von einer Aphis spec, von Cirsium oleraceum im optischen Querschnitt. Phasenkontrastaufnahme Dr. habil. Reumuth. Vergr. etwa 3000/1. - Abb. 4. Die Granulationen der Darmepithelzellen von Doralis mali in Hängetropfenkultur nach 24 Stdn. Vergr. etwa 1000/1. Abb.5. a. Die Darməpithelzellsymbionten von Mycodes persicae Sulzer, auf Pulstagar kultiviert. b. Auf Traubenmoste überimpft, entpuppen sie sich als Kahmhefen (Mycoderma). c. Die Symbionten in Sporulation. Die Sporen haben bereits die Ascei verlassen. Vergr. etwa 1000/1. - $\Lambda$ bb. 6 . Die bläschenförmigen Symbionten des eigentlichen Mycetoms einer Aphis von Cirsium oleraceum. Vergr. etwa 1000/1. 
9 unveränderten Samen in großen bedeckten Glasschalen auf feuchtem Fließpapier im Thermostaten bei $24^{\circ}$ zum Keimen ausgelegt. Schon nach 3 Tagen hatte unter den erstgenannten bei 6 Samen die Radicula die Testa durchstoßen. Die restlichen vier keimten auch später nicht mehr; sie verpilzten rasch. Von den frischen, mit Schleimhülle ausgelegten Samen hatten ebenfalls 6 die Testa gesprengt, einer hatte bereits eine Wurzel in Länge von $6 \mathrm{~mm}$ hervorgeschoben. Die übrigen 3 keimten auch hier nicht mehr aus, sondern wurden bald stark von Schimmelpilzen befallen. Die Schleimhülle der bereits ausgekeimten KakaoSamen wurde mit einer entsprechenden Menge dest. Wassers extrahiert. Der Auszug hemmte das Wurzelwachstum von Lepidium-Keimlingen um $50 \%$.

Auch hier keimen also die Kakao-Samen unmittelbar nach der Entnahme aus der Fruchthülle aus, unbeschadet der in ihren Schleimhüllen vorhandenen „Blasțokoline“ (s. Versuch Nr.3 in Tab. 2!). Selbst in den Schleimschichten ausgekeimter Kakao-Samen finden sich trotz einer gewissen Auslaugung noch Substanzen, die das Wurzelwachstum von Lepidium-Keimlingen stark hemmen, denn der nachträgliche Auszug aus den gekeimten Kakao-Samen entspricht etwá der Verdünnung 1:1 der oben mitgeteilten Versuche.

Am 5. Oktober wurde die letzte, ziemlich stark von Schimmelpilzen befallene Kakao-Frucht geöffnet, in der von den verpilzten Samen zwei und - von den unversehrten ebenfalls zwei ausgekeimt waren. Auffallend war, daß das Auskeimen von Kakao-Samen in der Frucht immer nur dann beobachtet wurde, wenn das Pericarp von Schimmelpilzen befallen war. Welche Umweltbedingung für den Samen sich damit verändert hat, läßt sich nicht ohne weiteres sagen. Möglich ist, daß z. B. die Sauerstoffversorgung wegen veränderter Durchlässigkeit der Fruchthülle gefördert wird. Daß eine relativ einfache Verschiebung der Umweltbedingungen die Keimung der Kakao-Samen auslösen muß, geht ja auch daraus hervor, daß die ungekeimten Samen der überreifen Früchte unmittelbar nach Entnahme aus den Früchten bei günstiger Wasserversorgung und optimaler Temperatur sofort auskeimen. Eine Verhinderung der Keimung in den Früchten liegt ohne $Z_{\text {weifel vor, }}$ aber sie beruht nicht auf den im Lepidium-Test nachweisbaren, das Wurzelwachstum hemmenden Substanzen. Die mit diesem Test erfaßten ,.Blasto- koline" sind auch keine Antagonisten der Wuchsstoffe, denn durch Zugabe von Wuchsstoffen (3Indolylessigsäure) zu den Schleimextrakten wird deren Hemmwirkung nicht geschwächt oder aufgehoben. Da Heteroauxin sowohl die Keimungsgeschwindigkeit als auch das Wurzelwachstum hemmt, addieren sich die beiden Hemmwirkungen. Es ist nicht ganz unwahrscheinlich, daß die im Schleim der Kakao-Samen nachweisbaren, das Wurzelwachstum hemmenden Substanzen Heteroauxin oder ähnliche Verbindungen sind, zumal beiläufig beobachtet wurde, daß Keimlinge, die auf verdünnten Schleimextrakten weiterwachsen, bei gehemmtem Wurzelwachstum Hypokotyle haben, die relativ stark gestreckt und manchmal länger als diejenigen der Kontrollen sind. Eine Auswertung der Extrakte in dieser Richtung hat jedoch nicht stattgefunden.

Eine biologische Bedeutung der in der Schleimschicht der Kakao-Samen gefundenen Hemmstoffe läßt sich nicht ohne weiteres angeben. Eine Möglichkeit wurde durch folgenden Versuch geprüft. Ein Same mit der Schleimhülle aus der letzten Frucht vom 5. Oktober wurde in eine Petrischale mit feuchtem Fließpapier gelegt und in Abständen von 1,2 und $3 \mathrm{~cm}$ in konzentrischen Kreisen von Lepidium-Samen umgeben und darauf 2 Tage bei $24^{\circ}$ in den Thermostaten gestellt. Nach dieser Zeit hatten die Lepidium-Samen des engsten Kreises eine durchschnittliche Wurzellänge von $1-2 \mathrm{~mm}$, in $2 \mathrm{~cm}$ Abstand waren die Wurzeln 2-8 $\mathrm{mm}$ und in $3 \mathrm{~cm}$ Abstand 10-16 mm lang. Der Kakao-Same hatte in dieser Zeit eine etwa $3 \mathrm{~mm}$ lange Keimwurzel getrieben. In der Nähe des Kakao-Samens wird also das Wachstum konkurrierender Samen anderer Arten hintenangehalten. Ob die Hemmstoffe aber tatsächlich auch unter normalen Verhältnissen dem Keimling als Waffe im Kampf ums Dasein dienen, kann dieser Versuch, in dem nicht mit Samen gearbeitet wurde, die an natürlichen Standorten des Kakao-Baumes konkurrieren, nicht entscheiden.

Die Ergebnisse der Versuche lassen sich in folgenden Sätzen zusammenfassen:

1. Wirklich keimungshemmende Stoffe konnten nur in den Schleimhüllen unreifer Kakao-Samen nachgewiesen werden.

2. Die Schleimextrakte der späteren Reifestadien wirken zwar noch stark hemmend auf das Wurzelwachstum bei Lepidium-Keimlingen, aber nicht auf das Auskeimen der Lepidium-Samen 
oder der Kakao-Samen selbst, obwohl die Hemmstoffe diese in höherer Konzentration umgeben, als in den Extrakten getestet wurde.

3. Das Auskeimen von Kakao-Samen in der Frucht setzt in späteren Reifestadien immer dann ein, wenn das Pericarp von Schimmelpilzen befallen worden ist, aber ohne daß ein im Lepidium-
Test nachweisbare Schwächung der Hemmstoffe eingetreten wäre. Die Faktoren, die das Auskeimen der Samen in gesunden reifen Früchten rerhindern, sind andere als die nachgewiesenen Hemmstoffe. Es ist an die Bildung flüchtiger Stoffwechselprodukte, z. B. an Äthylen, oder an unzureichenden Sauerstoffzutritt zu denken.

\title{
Studien über die Mycetom- und Darmsymbionten der Aphiden
}

\author{
Von Hugo Schanderl\%; Gertrud Lauff $\%$ und Helmut Becker
}

(Z. Naturforschg. 4 b, 50-53 [1949]; eingegangen am 16. Dezember 1948)

\begin{abstract}
Die aus den Aphidinen isolierten und teilweise kultivierten Symbionten wurden bisher von einem Teil der Forscher für Hefen, vom anderen Teil für Bakterien erklärt. Die Frage nach der Herkunft der Hefen konnte bei den Aphiden dahingehend geklärt werden, daß sie nicht aus den eigentlichen Mycetomen, sondern aus den Darmepithelzellen stammen. Die bläschenförmigen Granulationen der letzteren stellen nichts anderes als symbiontische Hefen dar, die unter strengsten aseptischen Bedingungen isoliert und in Kultur genommen werden konnten. Sie gehören zur Gattung Mycoderma. Der Mitteldarm der Aphidinen stellt also ein uraltes Symbioseorgan dar, das irgendwie mit der. eigenartigen Ernährungsweise der Blattläuse zusammenhängen muß.
\end{abstract}

$\mathrm{I}_{\mathrm{min}}^{\mathrm{m}}$ m hinteren Teil des Abdomens der Aphidinen befindet sich in unmittelbarer Nähe des Ovariums ein Organ, das von den alten Autoren mit dem Namen „Pseudovitellus" belegt wurde. Ihnen war bereits die Entstehung des Organes und das Einwandern rundlicher Gebilde (die nachher die Zellen des' „Pseudovitellus" anfüllen) in das Ei bekannt, ohne daß sie jedoch die wahre Natur des Vorganges erfaßten. $\mathrm{Pierantoni}{ }^{1}$ und $\mathrm{K}$. S s u l c erkannten als erste den Symbiosecharakter des Organes. K. Sulc hielt die Symbionten der Aphiden für Saccharomyceten und schlug deshalb die Bezeichnung ,Mycetom“ für den „Pseudovitellus" vor, die sich schnell einbürgerte. Pierantoni stellte die ersten Kulturversuche der Symbionten an. Er impfte auf Kartoffeln und erhielt durch Knospung sich vermehrende Organismen, die folglich als Hefen anzusprechen wären. P. Buchner studierte die Aphidensymbionten, enthielt sich aber eines Entscheides hinsichtlich ihrer systematischen Stellung. Peklo teilte seine Beobachtungen über die Symbionten von Pterocallis tiliae L, Aphis papaveris, Pemphigus und Schizoneura und über die Kultur derselben mit. Er deutete die Symbionten als eine Azotobacter-Art und vermutete, das das Mycetom funktionell den Wurzelknöllchen der Leguminosen entspräche. K levenhusen spricht ,von rundlichen Organis-

* (16) Geisenheim a. Rh., Botan. Inst. der Lehr- und Forschungsanstalt.

1 Die gesamte, bis 1930 (einschl.) erschienene dicsbezügl. Literatur ist in $\mathrm{P}$. B $\mathrm{u} c \mathrm{hne} \mathrm{n}$, Tier und Pflanze in Symbiose, 2. Aufl., Berlin 1930, angeführt.

2 Sch w a r t z, Untersuchungen über die Symbiose von Tieren mit Pilzen und Bakterien, Arch. Mikrobiol. 6, $369-461$ [19:5]. men, deren systematische Stellung ungeklärt ist". $\mathrm{U}$ i c h a n c o erhielt bei seinen Kulturversuchen wiederum ,hefeähnliche“ Organismen und glaubte weder für diese noch für die von anderen Forschern erhaltenen Mikroorganismen annehmen zu können, daß sie mit den wahren Symbionten identisch seien. Mit Buchner müssen wir ihm hierin beipflichten. Durch die Arbeiten von $\mathrm{Paillot}$ kam die Frage nach der ystematischen Zugehörigkeit der Symbionten anscheinend etwas weiter, indem sich dieser, wie Peklo, für die Bakteriennatur der Aphidensymbionten aussprach. Nach Paillot sollen die Symbionten Vertreter verschiedener Bakteriengattungen sein. Seine Feststellungen traf er lediglich an Hand von Ausstrichpräparaten, ohne Zuchtversuche unternommen zu haben. Solche betrieb Schwartz, aber ohne Erfolg. Die allgemeine Auffassung in dieser Frage kam in dessen Sammelreferat ${ }^{2}$ mit folgenden Worten zum Ausdruck: „Es besteht kaum ein $Z_{w}$ eifel, daß es sich hei den Symbionten der Blattläuse um Bakterien handelt". L. Haracsi ${ }^{3}$ fand im Zusammenhang mit Untersuchungen über die Speicheldrüsenentwicklung von Prociphilus bumeliae Schrk. innerhalb eines Syncytiums der sich entwickelnden Speicheldrüse neben den Kernen kleine kugel- und blasenförmige Einschlüsse, die er für den Saccharomyceten nächstrerwandte Symbionten hielt. Ähnlich wie Peklo hielten Toth, Wolsky und Batori ${ }^{4}$ die Mycetome der Aphidinen für Stätten der Luftstickstoffbindung.

3 Haracsi, Beiträge zur Biologie der Blattläuse, Diss. Sopron 1937 .

${ }_{4}$ Toth. Wolsky u. Batori. Stickstoffbindung aus der Luft bei den Aphiden und hei den Homopteren, Z.vergl. Physiol. 30, 67-7:3 [1942]. 